\title{
SOME PROPERTIES OF SOLUTIONS OF CERTAIN COMPLEX LINEAR DIFFERENTIAL EQUATIONS WITH MEROMORPHIC COEFFICIENTS*
}

\author{
BENHARRAT BELAÏDI $^{\dagger}$ AND ABDALLAH EL FARISSI ${ }^{\ddagger}$
}

\begin{abstract}
In this paper, we investigate the relationship between the solutions, their 1st and 2nd derivatives of some second order linear differential equations and meromorphic function of finite order. We obtain some precise estimates.
\end{abstract}

Key words. Linear differential equations, Meromorphic solutions, Order of growth, Exponent of convergence of zeros, Exponent of convergence of distinct zeros.

AMS subject classifications. 34M10, 30D35

1. Introduction and statement of results. Throughout this paper, we assume that the reader is familiar with the fundamental results and the standard notations of the Nevanlinna's value distribution theory (see [8], [10]). In addition, we will use $\lambda(f)$ and $\lambda(1 / f)$ to denote respectively the exponents of convergence of the zero-sequence and the pole-sequence of a meromorphic function $f, \rho(f)$ to denote the order of growth of $f, \bar{\lambda}(f)$ and $\bar{\lambda}(1 / f)$ to denote respectively the exponents of convergence of the sequence of distinct zeros and distinct poles of $f$.

Consider the second order linear differential equation

$$
f^{\prime \prime}+A_{1}(z) e^{P(z)} f^{\prime}+A_{0}(z) e^{Q(z)} f=0,
$$

where $P(z), Q(z)$ are nonconstant polynomials, $A_{1}(z), A_{0}(z)(\not \equiv 0)$ are entire functions such that $\rho\left(A_{1}\right)<\operatorname{deg} P(z), \rho\left(A_{0}\right)<\operatorname{deg} Q(z)$. Gundersen showed in [6, p. 419] that if $\operatorname{deg} P(z) \neq \operatorname{deg} Q(z)$, then every nonconstant solution of (1.1) is of infinite order. If $\operatorname{deg} P(z)=\operatorname{deg} Q(z)$, then (1.1) may have nonconstant solutions of finite order. For instance $f(z)=e^{z}+1$ satisfies $f^{\prime \prime}+e^{z} f^{\prime}-e^{z} f=0$.

In [2], Z. X. Chen and K. H. Shon have investigated the case when $\operatorname{deg} P(z)=$ $\operatorname{deg} Q(z)$ and have proved the following results:

Theorem A [2]. Let $A_{j}(z)(\not \equiv 0)(j=0,1)$ be meromorphic functions with $\rho\left(A_{j}\right)<1(j=0,1), a, b$ be complex numbers such that $a b \neq 0$ and $\arg a \neq \arg b$ or $a=c b(0<c<1)$. Then every meromorphic solution $f(z) \not \equiv 0$ of the equation

$$
f^{\prime \prime}+A_{1}(z) e^{a z} f^{\prime}+A_{0}(z) e^{b z} f=0
$$

has infinite order.

\footnotetext{
* Received June 20, 2007; accepted for publication May 22, 2008.

${ }^{\dagger}$ Department of Mathematics, Laboratory of Pure and Applied Mathematics, University of Mostaganem, B. P 227 Mostaganem, Algeria (belaidi@univ-mosta.dz; belaidi.benharrat@caramail.com).

$\ddagger$ Department of Mathematics, University of Mostaganem, B. P 227 Mostaganem, Algeria (el.farissi.abdallah@caramail.com).
} 
In the same paper, Z. X. Chen and K. H. Shon have investigated the fixed points of solutions, their 1st and 2nd derivatives and the differential polynomials and have obtained:

Theorem B [2]. Let $A_{j}(z)(j=0,1), a, b, c$ satisfy the additional hypotheses of Theorem A. Let $d_{0}, d_{1}, d_{2}$ be complex constants that are not all equal to zero.If $f(z) \not \equiv 0$ is any meromorphic solution of equation (1.2), then:

(i) $f, f^{\prime}, f^{\prime \prime}$ all have infinitely many fixed points and satisfy

$$
\bar{\lambda}(f-z)=\bar{\lambda}\left(f^{\prime}-z\right)=\bar{\lambda}\left(f^{\prime \prime}-z\right)=\infty,
$$

(ii) the differential polynomial

$$
g(z)=d_{2} f^{\prime \prime}+d_{1} f^{\prime}+d_{0} f
$$

has infinitely many fixed points and satisfies $\bar{\lambda}(g-z)=\infty$.

The main purpose of this paper is to study the relations between the solutions, their 1st and 2nd derivatives of the differential equation (1.1) and meromorphic functions of finite order. In fact we will prove the following results:

THEOREM 1.1. Let $P(z)=\sum_{i=0}^{n} a_{i} z^{i}$ and $Q(z)=\sum_{i=0}^{n} b_{i} z^{i}$ be nonconstant polynomials where $a_{i}, b_{i}(i=0,1, \ldots, n)$ are complex numbers, $a_{n} \neq 0, b_{n} \neq 0$ such that $a_{n}=c b_{n}(c>1)$ and $\operatorname{deg}(P-c Q)=m \geq 1$ and $A_{1}(z), A_{0}(z)(\not \equiv 0)$ be meromorphic functions with $\rho\left(A_{j}\right)<m(j=0,1)$. Let $d_{0}(z), d_{1}(z), d_{2}(z)$ be polynomials that are not all equal to zero, $\varphi(z) \not \equiv 0$ is a meromorphic function with finite order. If $f(z) \not \equiv 0$ is a meromorphic solution of $(1.1)$ with $\lambda(1 / f)<\infty$, then the differential polynomials $g(z)=d_{2} f^{\prime \prime}+d_{1} f^{\prime}+d_{0} f$ satisfy $\bar{\lambda}(g-\varphi)=\infty$.

REMARK 1.1. In the following Theorem 1.2, we remove the condition $\lambda(1 / f)<$ $\infty$.

Theorem 1.2. Suppose that $P(z), Q(z), A_{1}(z), A_{0}(z)$ satisfy the hypotheses of Theorem 1.1. If $\varphi(z) \not 0$ is a meromorphic function with finite order, then every meromorphic solution $f$ of $(1.1)$ satisfies $\bar{\lambda}(f-\varphi)=\bar{\lambda}\left(f^{\prime}-\varphi\right)=\bar{\lambda}\left(f^{\prime \prime}-\varphi\right)=\infty$.

2. Preliminary Lemmas. We need the following lemmas in the proofs of our theorems.

Lemma 2.1 [5]. Let $f$ be a transcendental meromorphic function of finite order $\rho$, let $\Gamma=\left\{\left(k_{1}, j_{1}\right),\left(k_{2}, j_{2}\right), \ldots,\left(k_{m}, j_{m}\right)\right\}$ denote a finite set of distinct pairs of integers that satisfy $k_{i}>j_{i} \geq 0$ for $i=1, \ldots, m$ and let $\varepsilon>0$ be a given constant. Then the following estimations hold:

(i) There exists a set $E_{1} \subset[0,2 \pi)$ that has linear measure zero, such that if $\psi \in$ $[0,2 \pi)-E_{1}$, then there is a constant $R_{1}=R_{1}(\psi)>1$ such that for all $z$ satisfying $\arg z=\psi$ and $|z| \geq R_{1}$ and for all $(k, j) \in \Gamma$, we have 


$$
\left|\frac{f^{(k)}(z)}{f^{(j)}(z)}\right| \leq|z|^{(k-j)(\rho-1+\varepsilon)} .
$$

(ii) There exists a set $E_{2} \subset(1, \infty)$ that has finite logarithmic measure $\operatorname{lm}\left(E_{2}\right)=$ $\int_{1}^{+\infty} \frac{\chi_{E_{2}}(t)}{t} d t$, where $\chi_{E_{2}}$ is the characteristic function of $E_{2}$, such that for all $z$ satisfying $|z| \notin E_{2} \cup[0,1]$ and for all $(k, j) \in \Gamma$, we have

$$
\left|\frac{f^{(k)}(z)}{f^{(j)}(z)}\right| \leq|z|^{(k-j)(\rho-1+\varepsilon)} .
$$

LEMma $2.2[2]$. Let $f(z)$ be a transcendental meromorphic function of order $\rho(f)=\rho<+\infty$. Then for any given $\varepsilon>0$, there exists a set $E_{3} \subset[0,2 \pi)$ that has linear measure zero, such that if $\psi_{1} \in[0,2 \pi) \backslash E_{3}$, then there is a constant $R_{2}=$ $R_{2}\left(\psi_{1}\right)>1$ such that for all $z$ satisfying $\arg z=\psi_{1}$ and $|z|=r \geq R_{2}$, we have

$$
\exp \left\{-r^{\rho+\varepsilon}\right\} \leq|f(z)| \leq \exp \left\{r^{\rho+\varepsilon}\right\} .
$$

Lemma 2.3. Let $P(z)=a_{n} z^{n}+\ldots+a_{0},\left(a_{n}=\alpha+i \beta \neq 0\right)$ be a polynomial with degree $n \geq 1$ and $A(z)(\not \equiv 0)$ be a meromorphic function with $\rho(f)<n$. Set $f(z)=A(z) e^{P(z)}, z=r e^{i \theta}, \delta(P, \theta)=\alpha \cos n \theta-\beta \sin n \theta$. Then for any given $\varepsilon>$ 0 , there exists a set $E_{4} \subset[0,2 \pi)$ that has linear measure zero, such that if $\theta \in$ $[0,2 \pi) \backslash\left(E_{4} \cup E_{5}\right)$, where $E_{5}=\{\theta \in[0,2 \pi): \delta(P, \theta)=0\}$ is a finite set, then for sufficiently large $|z|=r$, we have

(i) if $\delta(P, \theta)>0$, then

$$
\exp \left\{(1-\varepsilon) \delta(P, \theta) r^{n}\right\} \leq|f(z)| \leq \exp \left\{(1+\varepsilon) \delta(P, \theta) r^{n}\right\},
$$

(ii) if $\delta(P, \theta)<0$, then

$$
\exp \left\{(1+\varepsilon) \delta(P, \theta) r^{n}\right\} \leq|f(z)| \leq \exp \left\{(1-\varepsilon) \delta(P, \theta) r^{n}\right\}
$$

Proof. Set $f(z)=h(z) e^{(\alpha+i \beta) z^{n}}$, where $h(z)=A(z) e^{P_{n-1}(z)}, P_{n-1}(z)=P(z)-$ $(\alpha+i \beta) z^{n}$. Then $\rho(h)=\lambda<n$. By Lemma 2.2, for any given $\varepsilon(0<\varepsilon<n-\lambda)$, there is $E_{4} \subset[0,2 \pi)$ that has linear measure zero, such that if $\theta \in[0,2 \pi) \backslash\left(E_{4} \cup E_{5}\right)$, where $E_{5}=\{\theta \in[0,2 \pi): \delta(P, \theta)=0\}$, then there is a constant $R_{2}=R_{2}(\theta)>1$, such that, for all $z$ satisfying $\arg z=\theta$ and $r \geq R_{2}$, we have

$$
\exp \left\{-r^{\lambda+\varepsilon}\right\} \leq|h(z)| \leq \exp \left\{r^{\lambda+\varepsilon}\right\} .
$$

By $\left|e^{(\alpha+i \beta)\left(r e^{i \theta}\right)^{n}}\right|=e^{\delta(P, \theta) r^{n}}$ and (2.6), we have

$$
\exp \left\{\delta(P, \theta) r^{n}-r^{\lambda+\varepsilon}\right\} \leq|f(z)| \leq \exp \left\{\delta(P, \theta) r^{n}+r^{\lambda+\varepsilon}\right\} .
$$

By $\theta \notin E_{5}$ we see that: 
(i) if $\delta(P, \theta)>0$, then by $0<\lambda+\varepsilon<n$ and (2.7), we know that (2.4) holds for a sufficiently large $r$;

(ii) if $\delta(P, \theta)<0$, then by $0<\lambda+\varepsilon<n$ and (2.7), we know that (2.5) holds for a sufficiently large $r$.

Lemma 2.4 [4] . Let $A_{0}, A_{1}, \ldots, A_{k-1}, F \supsetneqq 0$ be finite order meromorphic functions. If $f$ is a meromorphic solution with $\rho(f)=\infty$ of the equation

$$
f^{(k)}+A_{k-1} f^{(k-1)}+\ldots+A_{1} f^{\prime}+A_{0} f=F,
$$

then $\bar{\lambda}(f)=\lambda(f)=\rho(f)=\infty$.

Lemma $2.5\left([7\right.$, p. 344] $)$. Let $f(z)=\sum_{n=0}^{\infty} a_{n} z^{n}$ be an entire function, $\mu(r)$ be the maximum term, i.e., $\mu(r)=\max \left\{\left|a_{n}\right| r^{n} ; n=0,1, \ldots\right\}$ and let $\nu_{f}(r)$ be the central index of $f$, i.e., $\nu_{f}(r)=\max \left\{m, \mu(r)=\left|a_{m}\right| r^{m}\right\}$. Then

$$
\nu_{f}(r)=r \frac{d}{d r} \log \mu(r)<[\log \mu(r)]^{2} \leq[\log M(r, f)]^{2},
$$

outside a set $E_{6} \subset(1,+\infty)$ of $r$ of finite logarithmic measure.

LEMma 2.6 (Wiman-Valiron, [7], [11]). Let $f(z)$ be a transcendental entire function, and let $z$ be a point with $|z|=r$ at which $|f(z)|=M(r, f)$. Then the estimation

$$
\frac{f^{(k)}(z)}{f(z)}=\left(\frac{\nu_{f}(r)}{z}\right)^{k}(1+o(1)) \quad(k \geq 1 \text { is an integer }),
$$

holds for all $|z|$ outside a set $E_{7}$ of $r$ of finite logarithmic measure.

Lemma $2.7([3])$. Suppose that $f(z)$ is a meromorphic function with $\rho(f)=$ $\beta<\infty$. Then for any given $\varepsilon>0$, there is a set $E_{8} \subset(1,+\infty)$ of finite logarithmic measure, such that

$$
|f(z)| \leq \exp \left\{r^{\beta+\varepsilon}\right\}
$$

holds for $|z|=r \notin[0,1] \cup E_{8}, r \rightarrow+\infty$.

LEMMA 2.8. Let $f(z)$ be a meromorphic function with $\rho(f)=\infty$ and the exponent of convergence of the poles of $f(z)$ is finite, $\lambda(1 / f)<\infty$. Let $d_{j}(z)(j=0,1,2)$ be polynomials that are not all equal to zero. Then

$$
g(z)=d_{2}(z) f^{\prime \prime}+d_{1}(z) f^{\prime}+d_{0}(z) f
$$

satisfies $\rho(g)=\infty$.

Proof. We suppose that $\rho(g)=\rho<\infty$ and then we obtain a contradiction. First we suppose that $d_{2}(z) \not \equiv 0$. Set $f(z)=w(z) / h(z)$, where $h(z)$ is canonical product (or polynomial) formed with the non-zero poles of $f(z), \lambda(h)=\rho(h)=\lambda(1 / f)=$ $\rho_{1}<\infty, w(z)$ is an entire function with $\rho(w)=\rho(f)=\infty$. We have 


$$
f^{\prime}(z)=\frac{w^{\prime} h-h^{\prime} w}{h^{2}} \text { and } f^{\prime \prime}(z)=\frac{w^{\prime \prime}}{h}-\frac{w h^{\prime \prime}}{h^{2}}-2 \frac{w^{\prime} h^{\prime}}{h^{2}}+2 \frac{\left(h^{\prime}\right)^{2} w}{h^{3}} .
$$

Hence

$$
\begin{gathered}
\frac{f^{\prime \prime}(z)}{f(z)}=\frac{w^{\prime \prime}}{w}-\frac{h^{\prime \prime}}{h}-2 \frac{w^{\prime} h^{\prime}}{w h}+2 \frac{\left(h^{\prime}\right)^{2}}{h^{2}}, \\
\frac{f^{\prime}(z)}{f(z)}=\frac{w^{\prime}}{w}-\frac{h^{\prime}}{h} .
\end{gathered}
$$

By Lemma 2.1 (ii), there exists a set $E_{1} \subset(1, \infty)$ that has finite logarithmic measure, such that, for all $z$ satisfying $|z| \notin E_{1} \cup[0,1]$, we have

$$
\left|\frac{h^{(j)}(z)}{h(z)}\right| \leq|z|^{j\left(\rho_{1}-1+\varepsilon\right)} \quad(j=1,2) .
$$

Substituting (2.16) into (2.14) and (2.15), we obtain for all $z$ satisfying $|z| \notin E_{1} \cup[0,1]$

$$
\begin{gathered}
\frac{f^{\prime \prime}(z)}{f(z)}=\frac{w^{\prime \prime}}{w}+O\left(z^{\alpha}\right) \frac{w^{\prime}}{w}+O\left(z^{\alpha}\right), \\
\frac{f^{\prime}(z)}{f(z)}=\frac{w^{\prime}}{w}+O\left(z^{\alpha}\right),
\end{gathered}
$$

where $\alpha(0<\alpha<\infty)$ is a constant and may be different at different places. Substituting (2.17) and (2.18) into (2.12), we have

$$
\begin{gathered}
d_{2}(z)\left(\frac{w^{\prime \prime}}{w}+O\left(z^{\alpha}\right) \frac{w^{\prime}}{w}+O\left(z^{\alpha}\right)\right) \\
+d_{1}(z)\left(\frac{w^{\prime}}{w}+O\left(z^{\alpha}\right)\right)+d_{0}(z)=\frac{g(z) h(z)}{w(z)} .
\end{gathered}
$$

It follows that

$$
\begin{gathered}
d_{2}(z) \frac{w^{\prime \prime}}{w}+\left(O\left(z^{\alpha}\right) d_{2}(z)+d_{1}(z)\right) \frac{w^{\prime}}{w}+O\left(z^{\alpha}\right) d_{2}(z) \\
+O\left(z^{\alpha}\right) d_{1}(z)+d_{0}(z)=\frac{g(z) h(z)}{w(z)} .
\end{gathered}
$$


Hence,

$$
d_{2}(z) \frac{w^{\prime \prime}}{w}+O\left(z^{m}\right) \frac{w^{\prime}}{w}+O\left(z^{m}\right)=\frac{g(z) h(z)}{w(z)}
$$

where $m(0<m<\infty)$ is some constant. By Lemma 2.6, there exists a set $E_{2} \subset$ $(1,+\infty)$ with logarithmic measure $\operatorname{lm}\left(E_{2}\right)<+\infty$ and we can choose $z$ satisfying $|z|=r \notin[0,1] \cup E_{2}$ and $|w(z)|=M(r, w)$, such that

$$
\frac{w^{(j)}(z)}{w(z)}=\left(\frac{\nu_{w}(r)}{z}\right)^{j}(1+o(1))(j=1,2) .
$$

Since $\rho(g)=\rho<\infty$ and $\rho(h)=\lambda(1 / f)=\rho_{1}<\infty$ by Lemma 2.7, there exists a set $E_{3}$ that has finite logarithmic measure, such that for all $z$ satisfying $|z|=r \notin$ $[0,1] \cup E_{3}$, we have

$$
|g(z)| \leq \exp \left\{r^{\rho+1}\right\},|h(z)| \leq \exp \left\{r^{\rho_{1}+1}\right\} .
$$

By Lemma 2.5, there is a set $E_{4} \subset(1,+\infty)$ that has finite logarithmic measure, such that for all $z$ satisfying $|z|=r \notin[0,1] \cup E_{4}$, we have

$$
\left|\nu_{w}(r)\right|<(\log M(r, w))^{2} .
$$

Since $\rho(w)=\varlimsup_{r \rightarrow+\infty} \frac{\log \nu_{w}(r)}{\log r}=+\infty$, there exists $\left\{r_{n}^{\prime}\right\}\left(r_{n}^{\prime} \rightarrow+\infty\right)$ such that

$$
\lim _{r_{n}^{\prime} \rightarrow+\infty} \frac{\log \nu_{w}\left(r_{n}^{\prime}\right)}{\log r_{n}^{\prime}}=+\infty
$$

Set the logarithmic measure of $E_{1} \cup E_{2} \cup E_{3} \cup E_{4}, \operatorname{lm}\left(E_{1} \cup E_{2} \cup E_{3} \cup E_{4}\right)=\gamma<+\infty$, then there exists a point $r_{n} \in\left[r_{n}^{\prime},(\gamma+1) r_{n}^{\prime}\right]-E_{1} \cup E_{2} \cup E_{3} \cup E_{4}$. From

$$
\frac{\log \nu_{w}\left(r_{n}\right)}{\log r_{n}} \geq \frac{\log \nu_{w}\left(r_{n}^{\prime}\right)}{\log \left((\gamma+1) r_{n}^{\prime}\right)}=\frac{\log \nu_{w}\left(r_{n}^{\prime}\right)}{\left[1+\frac{\ln (\gamma+1)}{\log r_{n}^{\prime}}\right] \log r_{n}^{\prime}}
$$

it follows that

$$
\lim _{r_{n} \rightarrow+\infty} \frac{\log \nu_{w}\left(r_{n}\right)}{\log r_{n}}=+\infty .
$$

Then for a given arbitrary large $\beta>2\left(\rho_{1}+\rho+m+3\right)$,

$$
\nu_{w}\left(r_{n}\right) \geq r_{n}^{\beta}
$$

holds for sufficiently large $r_{n}$. Now we take point $z_{n}$ satisfying $\left|z_{n}\right|=r_{n}$ and $w\left(z_{n}\right)=$ $M\left(r_{n}, w\right)$, by $(2.21)$ and $(2.22)$, we get 


$$
\begin{aligned}
\left|d_{2}\left(z_{n}\right)\right|\left(\frac{\nu_{w}\left(r_{n}\right)}{r_{n}}\right)^{2} & |1+o(1)| \leq 2 L r_{n}^{m}\left(\frac{\nu_{w}\left(r_{n}\right)}{r_{n}}\right)|1+o(1)| \\
& +\left|\frac{g\left(z_{n}\right) h\left(z_{n}\right)}{w\left(z_{n}\right)}\right|,
\end{aligned}
$$

where $L>0$ is some constant. By Lemma 2.5 and (2.28), we get

$$
M\left(r_{n}, w\right)>\exp \left(r_{n}^{\frac{\beta}{2}}\right) .
$$

Hence by $(2.23),(2.30)$ as $r_{n} \rightarrow+\infty$

$$
\frac{\left|g\left(z_{n}\right) h\left(z_{n}\right)\right|}{M\left(r_{n}, w\right)} \rightarrow 0
$$

holds. By (2.28), (2.29), (2.31), we get

$$
\left|d_{2}\left(z_{n}\right)\right| r_{n}^{\beta} \leq\left|d_{2}\left(z_{n}\right)\right| \nu_{w}\left(r_{n}\right) \leq 2 L K r_{n}^{m+1},
$$

where $K>0$ is some constant. This is a contradiction by $\beta>2\left(\rho_{1}+\rho+m+3\right)$. Hence $\rho(g)=\infty$.

Now suppose $d_{2} \equiv 0, d_{1} \equiv 0$. Using a similar reasoning as above we get a contradiction. Hence $\rho(g)=\infty$.

Finally, if $d_{2} \equiv 0, d_{1} \equiv 0, d_{0} \equiv 0$, then we have $g(z)=d_{0}(z) f(z)$ and by $d_{0}$ is a polynomial, then we get $\rho(g)=\infty$.

LEMma 2.9. Let $P(z)=\sum_{i=0}^{n} a_{i} z^{i}$ and $Q(z)=\sum_{i=0}^{n} b_{i} z^{i}$ be nonconstant polynomials where $a_{i}, b_{i}(i=0,1, \ldots, n)$ are complex numbers, $a_{n} \neq 0, b_{n} \neq 0$ such that $a_{n}=c b_{n}$ $(c>1)$. We denote index sets by

$$
\begin{gathered}
\Lambda_{1}=\{0, Q\}, \\
\Lambda_{2}=\{0, Q, P, 2 Q, P+Q\} .
\end{gathered}
$$

(i) If $H_{j}\left(j \in \Lambda_{1}\right)$ and $H_{P} \not \equiv$ are all meromorphic functions of orders that are less than $n$, setting $\Psi_{1}(z)=\sum_{j \in \Lambda_{1}} H_{j}(z) e^{j}$, then $\Psi_{1}(z)+H_{P} e^{P} \not \equiv 0$;

(ii) If $H_{j}\left(j \in \Lambda_{2}\right)$ and $H_{2 P} \not 0$ are all meromorphic functions of orders that are less than $n$, setting $\Psi_{2}(z)=\sum_{j \in \Lambda_{2}} H_{j}(z) e^{j}$, then $\Psi_{2}(z)+H_{2 P} e^{2 P} \not 0$.

(iii) Let $\Psi_{10}(z), \Psi_{11}(z)$ have the form of $\Psi_{2}(z)$ which is defined as in (ii) and $H_{2 P} \equiv 0$ are all meromorphic functions of orders that are less than $n, \varphi(z) \not 0$ is a meromorphic functions with finite order. Then 


$$
\frac{\varphi^{\prime}(z)}{\varphi(z)} \Psi_{11}(z)++\Psi_{10}(z)+H_{2 P} e^{2 P} \not 0 .
$$

Proof. The proof of $(i)$ and $(i i)$ are similar, we prove only $(i i)$.

Suppose $a_{n}=c b_{n}(c>1)$. Set $\rho\left(H_{0}\right)=\beta<n$. By Lemma 2.2, for any given $\varepsilon$ $\left(0<\varepsilon<\min \left(\frac{c-1}{5 c}, n-\beta\right)\right)$, there is a set $E_{1}$ that has linear measure zero such that if $\arg z=\theta \in[0,2 \pi) \backslash E_{1}$, then there is $R=R(\theta)>1$ such that for all $z$ satisfying $\arg z=\theta$ and $|z|=r>R$, we have

$$
\left|H_{0}(z)\right| \leq \exp \left\{r^{\beta+\varepsilon}\right\} .
$$

By Lemma 2.3, there exists a ray $\arg z=\theta \in[0,2 \pi) \backslash E_{1} \cup E_{2} \cup E_{0}, E_{2}, E_{0} \subset[0,2 \pi)$ being defined as in Lemma 2.3, $E_{2}$ having linear measure zero, $E_{0}$ being a finite set, such that $\delta(Q, \theta)>0$

$$
\begin{gathered}
\delta(P, \theta)=c \delta(Q, \theta)>0, \quad \delta(2 P, \theta)=2 c \delta(Q, \theta)>0, \\
\delta(P+Q, \theta)=(1+c) \delta(Q, \theta)>0, \delta(2 Q, \theta)=2 \delta(Q, \theta)>0 .
\end{gathered}
$$

And for the above $\varepsilon$, we have for sufficiently large $|z|=r$

$$
\begin{gathered}
\left|H_{2 P} e^{2 P}\right| \geq \exp \left\{(1-\varepsilon) 2 c \delta(Q, \theta) r^{n}\right\}, \\
\left|H_{P} e^{P}\right| \leq \exp \left\{(1+\varepsilon) c \delta(Q, \theta) r^{n}\right\}, \\
\left|H_{P+Q} e^{P+Q}\right| \leq \exp \left\{(1+\varepsilon)(1+c) \delta(Q, \theta) r^{n}\right\}, \\
\left|H_{2 Q} e^{2 Q}\right| \leq \exp \left\{(1+\varepsilon) 2 \delta(Q, \theta) r^{n}\right\}, \\
\left|H_{Q} e^{Q}\right| \leq \exp \left\{(1+\varepsilon) \delta(Q, \theta) r^{n}\right\} .
\end{gathered}
$$

If $\Psi_{2}(z)+H_{2 P} e^{2 P} \equiv 0$, then by $(2.33)-(2.38)$, we have

$$
\begin{gathered}
\exp \left\{(1-\varepsilon) 2 c \delta(Q, \theta) r^{n}\right\} \leq\left|H_{2 P} e^{2 P}\right| \leq \exp \left\{r^{\beta+\varepsilon}\right\}+\exp \left\{(1+\varepsilon) \delta(Q, \theta) r^{n}\right\} \\
+\exp \left\{(1+\varepsilon) c \delta(Q, \theta) r^{n}\right\}+\exp \left\{(1+\varepsilon) 2 \delta(Q, \theta) r^{n}\right\} \\
+\exp \left\{(1+\varepsilon)(1+c) \delta(Q, \theta) r^{n}\right\} \leq \exp \left\{r^{\beta+\varepsilon}\right\} \\
+4 \exp \left\{(1+\varepsilon)(1+c) \delta(Q, \theta) r^{n}\right\}
\end{gathered}
$$


By $\beta+\varepsilon<n$ and $0<\varepsilon<\frac{c-1}{5 c}$, we have as $r \rightarrow+\infty$

$$
\begin{gathered}
\frac{\exp \left\{r^{\beta+\varepsilon}\right\}}{\exp \left\{(1-\varepsilon) 2 c \delta(Q, \theta) r^{n}\right\}} \rightarrow 0, \\
\frac{\exp \left\{(1+\varepsilon)(1+c) \delta(Q, \theta) r^{n}\right\}}{\exp \left\{(1-\varepsilon) 2 c \delta(Q, \theta) r^{n}\right\}} \rightarrow 0 .
\end{gathered}
$$

By $(2.39)-(2.41)$, we get $1 \leq 0$. This is a contradiction, hence $\Psi_{2}(z)+H_{2 P} e^{2 P} \not 0$. (iii) By $\rho(\varphi)<\infty$ and Lemma 2.1, for any given $\varepsilon>0$, there exists a set $E \subset[0,2 \pi)$ that has linear measure zero, such that if $\theta \in[0,2 \pi)-E$, then there is a constant $R=R(\theta)>1$ such that for all $z$ satisfying $\arg z=\theta$ and $|z| \geq R$, we have

$$
\left|\frac{\varphi^{\prime}(z)}{\varphi(z)}\right| \leq|z|^{(\rho(\varphi)-1+\varepsilon)} .
$$

It follows that on the ray $\arg z=\theta \in[0,2 \pi)-E$,

$$
\frac{\varphi^{\prime}(z)}{\varphi(z)} H_{j}(z) e^{j} \quad\left(j \in \Lambda_{2}\right),
$$

keep the properties of $H_{j}(z) e^{j}\left(j \in \Lambda_{2}\right)$ which are defined as in (2.33), (2.35)-(2.38). By using similar reasoning to that in the proof of $(i i)$, the proof of $(i i i)$ can be completed.

Lemma $2.10[1]$. Let $P_{j}(z)=\sum_{i=0}^{n} a_{i, j} z^{i}(j=0, \ldots, k-1)$ be nonconstant polynomials where $a_{0, j}, \ldots, a_{n, j}(j=0,1, \ldots, k-1)$ are complex numbers such that $a_{n, j} a_{n, 0} \neq 0$ $(j=1, \ldots, k-1)$, let $A_{j}(z)(\neq 0)(j=0, \ldots, k-1)$ be meromorphic functions. Suppose that $a_{n, j}=c a_{n, 0}(c>1)$ and $\operatorname{deg}\left(P_{j}-c P_{0}\right)=m \geq 1(j=1, \ldots, k-1)$, $\rho\left(A_{j}\right)<m(j=0, \ldots, k-1)$. Then every meromorphic solution $f(z) \not \equiv 0$ of the equation

$$
f^{(k)}+A_{k-1}(z) e^{P_{k-1}(z)} f^{(k-1)}+\ldots+A_{1}(z) e^{P_{1}(z)} f^{\prime}+A_{0}(z) e^{P_{0}(z)} f=0,
$$

where $k \geq 2$, is of infinite order.

3. Proof of Theorem 1.1. Suppose that $f(z) \not 0$ is a meromorphic solution of equation (1.1) with $\lambda(1 / f)<\infty$. Then by Lemma 2.10, we have $\rho(f)=\infty$. First we suppose that $d_{2}(z) \not 0$. Set $w=d_{2} f^{\prime \prime}+d_{1} f^{\prime}+d_{0} f-\varphi$, then by Lemma 2.8 we have $\rho(w)=\rho(g)=\rho(f)=\infty$. In order to prove $\bar{\lambda}(g-\varphi)=\infty$, we need to prove $\bar{\lambda}(w)=\infty$. Substituting $f^{\prime \prime}=-A_{1} e^{P} f^{\prime}-A_{0} e^{Q} f$ into $w$, we get

$$
w=\left(d_{1}-d_{2} A_{1} e^{P}\right) f^{\prime}+\left(d_{0}-d_{2} A_{0} e^{Q}\right) f-\varphi .
$$

Differentiating both sides of equation (3.1) and replacing $f^{\prime \prime}$ with $f^{\prime \prime}=-A_{1} e^{P} f^{\prime}-$ $A_{0} e^{Q} f$, we obtain 


$$
\begin{aligned}
w^{\prime}= & {\left[d_{2} A_{1}^{2} e^{2 P}-\left(\left(d_{2} A_{1}\right)^{\prime}+P^{\prime} d_{2} A_{1}+d_{1} A_{1}\right) e^{P}-d_{2} A_{0} e^{Q}+d_{0}+d_{1}^{\prime}\right] f^{\prime} } \\
& +\left[d_{2} A_{0} A_{1} e^{P+Q}-\left(\left(d_{2} A_{0}\right)^{\prime}+Q^{\prime} d_{2} A_{0}+d_{1} A_{0}\right) e^{Q}+d_{0}^{\prime}\right] f-\varphi^{\prime}
\end{aligned}
$$

Set

$$
\begin{gathered}
\alpha_{1}=d_{1}-d_{2} A_{1} e^{P}, \alpha_{0}=d_{0}-d_{2} A_{0} e^{Q}, \\
\beta_{1}=d_{2} A_{1}^{2} e^{2 P}-\left(\left(d_{2} A_{1}\right)^{\prime}+P^{\prime} d_{2} A_{1}+d_{1} A_{1}\right) e^{P}-d_{2} A_{0} e^{Q}+d_{0}+d_{1}^{\prime}, \\
\beta_{0}=d_{2} A_{0} A_{1} e^{P+Q}-\left(\left(d_{2} A_{0}\right)^{\prime}+Q^{\prime} d_{2} A_{0}+d_{1} A_{0}\right) e^{Q}+d_{0}^{\prime} .
\end{gathered}
$$

Then we have

$$
\begin{gathered}
\alpha_{1} f^{\prime}+\alpha_{0} f=w+\varphi, \\
\beta_{1} f^{\prime}+\beta_{0} f=w^{\prime}+\varphi^{\prime} .
\end{gathered}
$$

Set

$$
h=\alpha_{1} \beta_{0}-\alpha_{0} \beta_{1} .
$$

Case 1: If $h(z) \equiv 0$, then by $(3.6),(3.7)$ we have

$$
\alpha_{1} w^{\prime}-\beta_{1} w=-\left(\alpha_{1} \varphi^{\prime}-\beta_{1} \varphi\right)=-\varphi\left(\alpha_{1} \frac{\varphi^{\prime}}{\varphi}-\beta_{1}\right) .
$$

By Lemma $2.9(i)-(i i)$ we have $\alpha_{1} \not \equiv 0, \beta_{1} \not \equiv 0$, and by $\varphi(z) \not \equiv 0$, Lemma 2.9 (iii), we obtain

$$
-\left(\alpha_{1} \varphi^{\prime}-\beta_{1} \varphi\right)=-\varphi\left(\alpha_{1} \frac{\varphi^{\prime}}{\varphi}-\beta_{1}\right) \not \equiv 0 .
$$

From (3.8), (3.9) and Lemma 2.4, we get $\bar{\lambda}(w)=\rho(w)=\infty$. Hence $\bar{\lambda}(g-\varphi)=\infty$. Case 2: If $h(z) \not 0$ then by (3.6), (3.7) we obtain

$$
f=\frac{\alpha_{1}\left(w^{\prime}+\varphi^{\prime}\right)-\beta_{1}(w+\varphi)}{h} .
$$


Substituting (3.10) into (1.1), we obtain

$$
\begin{gathered}
\left(\frac{\alpha_{1}\left(w^{\prime}+\varphi^{\prime}\right)-\beta_{1}(w+\varphi)}{h}\right)^{\prime \prime}+A_{1} e^{P}\left(\frac{\alpha_{1}\left(w^{\prime}+\varphi^{\prime}\right)-\beta_{1}(w+\varphi)}{h}\right)^{\prime} \\
+A_{0} e^{Q}\left(\frac{\alpha_{1}\left(w^{\prime}+\varphi^{\prime}\right)-\beta_{1}(w+\varphi)}{h}\right)=0
\end{gathered}
$$

By (3.11), we get

$$
\begin{aligned}
& \left(\frac{\alpha_{1} w^{\prime}-\beta_{1} w}{h}\right)^{\prime \prime}+A_{1} e^{P}\left(\frac{\alpha_{1} w^{\prime}-\beta_{1} w}{h}\right)^{\prime}+A_{0} e^{Q}\left(\frac{\alpha_{1} w^{\prime}-\beta_{1} w}{h}\right) \\
= & -\left[\left(\frac{\alpha_{1} \varphi^{\prime}-\beta_{1} \varphi}{h}\right)^{\prime \prime}+A_{1} e^{P}\left(\frac{\alpha_{1} \varphi^{\prime}-\beta_{1} \varphi}{h}\right)^{\prime}+A_{0} e^{Q}\left(\frac{\alpha_{1} \varphi^{\prime}-\beta_{1} \varphi}{h}\right)\right] .
\end{aligned}
$$

By (3.12), we obtain

$$
\frac{\alpha_{1}}{h} w^{\prime \prime \prime}+\Phi_{2} w^{\prime \prime}+\Phi_{1} w^{\prime}+\Phi_{0} w=F
$$

where $\Phi_{0}(z), \Phi_{1}(z)$ and $\Phi_{2}(z)$ are meromorphic functions with $\rho\left(\Phi_{0}\right) \leq n, \rho\left(\Phi_{1}\right) \leq$ $n, \rho\left(\Phi_{2}\right) \leq n$ and

$$
F=-\left[\left(\frac{\alpha_{1} \varphi^{\prime}-\beta_{1} \varphi}{h}\right)^{\prime \prime}+A_{1} e^{P}\left(\frac{\alpha_{1} \varphi^{\prime}-\beta_{1} \varphi}{h}\right)^{\prime}+A_{0} e^{Q}\left(\frac{\alpha_{1} \varphi^{\prime}-\beta_{1} \varphi}{h}\right)\right]
$$

By $\alpha_{1} \varphi^{\prime}-\beta_{1} \varphi \not 0, \rho\left(\frac{\alpha_{1} \varphi^{\prime}-\beta_{1} \varphi}{h}\right)<\infty$ and Lemma 2.10 we know that $F \not 0$. Hence by $h \not 0, \alpha_{1} \not 0$ and Lemma 2.4 , we obtain $\bar{\lambda}(w)=\rho(w)=\infty$. Hence $\bar{\lambda}(g-\varphi)=\infty$.

Now suppose $d_{2} \equiv 0, d_{1} \neq 0$. Using a similar reasoning as above we get $\bar{\lambda}(w)=$ $\rho(w)=\infty$. Hence $\bar{\lambda}(g-\varphi)=\infty$.

Finally, if $d_{2} \equiv 0, d_{1} \equiv 0, d_{0} \equiv 0$, then we have $w=d_{0} f-\varphi, \rho(w)=\infty$, $\bar{\lambda}(w)=\bar{\lambda}\left(d_{0} f-\varphi\right)$. Substituting

$$
f=\frac{w}{d_{0}}+\frac{\varphi}{d_{0}}, f^{\prime}=\left(\frac{w}{d_{0}}\right)^{\prime}+\left(\frac{\varphi}{d_{0}}\right)^{\prime}, f^{\prime \prime}=\left(\frac{w}{d_{0}}\right)^{\prime \prime}+\left(\frac{\varphi}{d_{0}}\right)^{\prime \prime}
$$

into equation (1.1), we obtain

$$
\left(\frac{w}{d_{0}}\right)^{\prime \prime}+A_{1} e^{P}\left(\frac{w}{d_{0}}\right)^{\prime}+A_{0} e^{Q} \frac{w}{d_{0}}=-\left(\left(\frac{\varphi}{d_{0}}\right)^{\prime \prime}+A_{1} e^{P}\left(\frac{\varphi}{d_{0}}\right)^{\prime}+A_{0} e^{Q} \frac{\varphi}{d_{0}}\right) .
$$


By $\varphi(z)$ being a meromorphic function of finite order and $d_{0}(z)$ is a polynomial, then $\frac{\varphi(z)}{d_{0}(z)}$ has finite order and by Lemma 2.10, we have

$$
\left(\frac{\varphi}{d_{0}}\right)^{\prime \prime}+A_{1} e^{P}\left(\frac{\varphi}{d_{0}}\right)^{\prime}+A_{0} e^{Q} \frac{\varphi}{d_{0}} \not 0
$$

Hence by Lemma 2.4, we have $\bar{\lambda}\left(\frac{w}{d_{0}}\right)=\rho\left(\frac{w}{d_{0}}\right)=\infty$ ( $d_{0}$ is a polynomial). Then $\bar{\lambda}(w)=\infty$, i.e., $\bar{\lambda}\left(d_{0} f-\varphi\right)=\infty$.

4. Proof of Theorem 1.2. Suppose that $f(z) \not 0$ is a meromorphic solution of equation (1.1). Then by Theorem 2.10 we have $\rho(f)=\rho\left(f^{\prime}\right)=\rho\left(f^{\prime \prime}\right)=\infty$. Since $\rho(\varphi)<\infty$, then $\rho(f-\varphi)=\rho\left(f^{\prime}-\varphi\right)=\rho\left(f^{\prime \prime}-\varphi\right)=\infty$. By using similar reasoning to that in the proof of Theorem 1.1, the proof of Theorem 1.2 can be completed.

\section{REFERENCES}

[1] B. BElaïDI, On the meromorphic solutions of linear differential equations, J. Syst. Sci. and Complexity, 20 (2007), pp. 41-46.

[2] Z.-X. Chen And K. H. Shon, On the growth and fixed points of solutions of second order differential equations with meromorphic coefficients, Acta Mathematica Sinica Engl. Ser., 21 (2005), pp. 753-764.

[3] Z.-X. Chen AND C.-C. YANG, Some oscillation theorems for linear differential equations with meromorphic coefficients, Southeast Asian Bull. Math., 23 (1999), pp. 409-417.

[4] Z.-X. Chen, Zeros of meromorphic solutions of higher order linear differential equations, Analysis, 14 (1994), pp. 425-438.

[5] G. G. Gundersen, Estimates for the logarithmic derivative of a meromorphic function, plus similar estimates, J. London Math. Soc., (2) 37 (1988), pp. 88-104.

[6] G. G. Gundersen, Finite order solutions of second order linear differential equations, Trans. Amer. Math. Soc., 305 (1988), pp. 415-429.

[7] W. K. Hayman, The local growth of power series: a survey of the Wiman-Valiron method, Canad. Math. Bull., 17 (1974), pp. 317-358.

[8] W. K. Hayman, Meromorphic functions, Clarendon Press, Oxford, 1964.

[9] K. H. Kwon, Nonexistence of finite order solutions of certain second order linear differential equations, Kodai Math. J., 19 (1996), pp. 378-387.

[10] R. Nevanlinna, Eindeutige Analytische Funktionen, Zweite Auflage. Reprint. Die Grundlehren der mathematischen Wissenschaften, Band 46. Springer-Verlag, Berlin-New York, 1974.

[11] G. VAlinon, Lectures on the General Theory of Integral Functions, translated by E. F. Collingwood, Chelsea, New York, 1949. 